\title{
Pyrolytic decomposition of solid propellants
}

\author{
R.A. Pesce-Rodriguez and R.A. Fifer
}

US Army Ballistic Research Laboratory, Aberdeen Proving Ground, MD 21005-5066

\begin{abstract}
Pyrolysis - gas chromatography - Fourier transform infrared (P-GC-FTIR) spectroscopy has been used to examine the pyrolysis product distributions of a composite propellant formulation series. The series was formulated with the oxidizers $H M X$ and RDX, the polymers GAP, HTPB, BAMO/AMMO, and BAMO/THF, and the plasticizers BTTN and TMETN. Trends in product distribution as a function of formulation, as well as correlations between radiative ignition times and pyrolysis products were identified. The most noteworthy correlations observed were between the amounts of permanent gases and amides produced and go/no-go ignition time. In general, pyrolysis product distribution were found to be most strongly affected by the presence and type of plasticizer.
\end{abstract}

\section{INTRODUCTION}

The objective of this investigation was to first identify the pyrolysis products of a series of nitramine-based propellants and then look for correlations between those pyrolysis products and radiative ignition times. Such correlations could be used to the identify "desirable" pyrolysis products and suggest possible additives or ingredients for control and customization of ignition times. Correlations could also be used for bench-scale screening tests of new propellant formulations. On a less applied level, the information provided by this type of investigation contributes to the elucidation of mechanisms and reactions taking place near the boundry between the condensed and vapor phases during ignition and combustion of solid propellants. The feature that distinguishes this type of investigation from most investigations of nitramine decompostion (refs. 1-3, reviews) is that through the use of gas chromatography - Fourier transform infrared (GC-FTIR) spectroscopy, the levels of large species, as well as permanent gases can be determined.

\section{EXPERIMENTAL}

Samples were placed in quartz capillary tubes and held in place with glass wool. The tube was then inserted into a coil-type pyroprobe. The probe was inserted into a heated interface which was continuously being swept with carrier gas. After one minute in the interface the sample was flash heated to the pyrolysis temperature and held at that temperature for $20 \mathrm{~s}$. The pyrolysis products then passed through the splitless injector into the capillary column, which separated the products for detection and identification. As each component eluted from the capillary column, it passed through a light pipe in the beam of an interferometer for spectral analysis by FTIR spectroscopy. Pyrolytic, chromatographic, and spectroscopic conditions are given as follows: Approximately $1 \mathrm{mg}$ of each sample was pyrolyzed using a Chemical Data Systems (CDS) Model 122 Pyroprobe connected via a heated CDS interface chamber to the splitless injector of a Hewlett Packard 5965 GC equipped with a capillary column and liquid nitrogen cooled mercury cadmium telluride (MCT) detector (Hewlett Packard Model 5965A infrared detector).

Chromatographic and spectroscopic conditions: Quadrex capillary column, $0.32 \mathrm{~mm} \times 25 \mathrm{~m}$ $x 3 \mu \mathrm{m}$ OV $-17 \mathrm{film}$; oven program: $50^{\circ} \mathrm{C}$ for $3 \mathrm{~min}$, then $50^{\circ} \mathrm{C}$ to $200^{\circ} \mathrm{C}$ at $10 \mathrm{deg} / \mathrm{min}$; injector and interface chamber held at $100^{\circ} \mathrm{C}$. FTIR conditions: transfer lines and light pipe held at $200^{\circ} \mathrm{C}$; three interferograms $/ \mathrm{sec}$ at $8 \mathrm{~cm}^{-1}$. Pyrolysis set temperature: $400^{\circ} \mathrm{C}$ for RDX formulations; $500^{\circ} \mathrm{C}$ for $\mathrm{HMX}$ formulations. Actual temperatures in the quartz tubes were $150-200^{\circ} \mathrm{C}$ lower than the set temperatures. 
The composition of each formulation examined is given in Table 1. Samples and performance measurements were kindly provided by Dr. Rena Yee, Naval Weapons Center, China Lake, California. The ingredients used were RDX, HMX, GAP (glycidyl azide polymer), BAMO/THF (3,3-bis-azido-methyl oxetane/tetrahydrofuran copolymer), BAMO/AMMO (BAMO/3,3-bis-azidomethyl-3-methyl oxetane copolymer), HTPB (hydroxy-terminated polybutadiene), TMETN (trimethylolethane trinitrate), and BTTN (1,2,4-butane trinitrate).

TABLE 1: Composition of Propellant Formulation

\begin{tabular}{|c|c|c|c|c|c|c|}
\hline Sample & RDX & $\begin{array}{r}\mathrm{HMX} \\
\text { (Co }\end{array}$ & $\begin{array}{l}\text { Polymer } \\
\text { position }\end{array}$ & $\begin{array}{r}\text { Type } \\
\text { (n weight-\%) }\end{array}$ & Plasticizer & Type \\
\hline 4 & 74.8 & & 6.3 & GAP & 18.9 & TMETN \\
\hline 8 & 68.4 & & 31.6 & GAP & & \\
\hline 9 & 65.9 & & 11.5 & GAP & 22.6 & BTTN \\
\hline 14 & & 69.7 & 30.3 & GAP & & \\
\hline 15 & & & 50.0 & GAP & 50.0 & BTTN \\
\hline 16 & 75.0 & & 25.0 & HTPB & & \\
\hline 17 & & 76.0 & 24.0 & HTPB & & \\
\hline 18 & & & 50.0 & GAP & 50.0 & TMETN \\
\hline 19 & 65.0 & & 17.5 & GAP & 17.5 & BTTN \\
\hline 20 & & 66.3 & 16.8 & GAP & 16.9 & BTTN \\
\hline 21 & & 67.6 & 16.2 & BAMO/THF & 16.2 & BTTN \\
\hline 22 & & 68.2 & 15.9 & BAMO/AMMO & 15.9 & TMETN \\
\hline 23 & & 68.6 & 15.7 & BAMO/THF & 15.7 & TMETN \\
\hline 24 & & 68.3 & 15.8 & GAP & 15.9 & TMETN \\
\hline 25 & 67.1 & & 16.6 & GAP & 16.4 & TMETN \\
\hline
\end{tabular}

\section{RESULTS}

Pyrolysis product distributions

The primary experimental data obtained from these experiments are GC peak areas. Retention times and FTIR spectra aid in the identification of pyrolysis products. Based on such information, product distributions for 15 different propellant formulations were determined. Pyrolyses at $1000^{\circ} \mathrm{C}$ were also performed, but are not discussed here due to space limitations. Quantification of pyrolysis products was based on GC peak areas and is reported in area percent (Table 2). Exceptions to this are the individual permanent gas products which are not readily quantified by GC-peak area because the elute within a few seconds of each other and appear as a single GC peak. For this reason, individual permanent gas quantities were calculated from FTIR absorbance and are given in normalized absorbance units (Table 3 ). To calculate these normalized absorbance values, all FTIR spectra under the permanent gas peak were first summed to yield a simgle spectrum. The absorbance of the largest band for each of the gases in the spectrum was then divided by the sum of the absorbances of the largest band for each gas. The bands chosen for each gas are given as follows: $\mathrm{CH}_{4}, 3016 \mathrm{~cm}^{-1} ; \mathrm{CH}_{2} \mathrm{O}, 2084 \mathrm{~cm}^{-1} ; \mathrm{CO}_{2}, 2363 \mathrm{~cm}^{-1} ; \mathrm{N}_{2} \mathrm{O}, 2238$ $\mathrm{cm}^{-1}$; CO, $2111 \mathrm{~cm}^{-1}$; and NO, $1912 \mathrm{~cm}^{-1}$. All reported values are uncalibrated, relative quantities that are only used to identify variations in pyrolysis product distributions. Magnitudes of absorbance, as well as GC peak areas, for different compounds are not comparable due to differences in infrared absorption coefficients.

Although the data reported here represent one of the most comprehensive investigations of pyrolysis product distribution for propellant formulations to date, several products are notably absent. Most of these products (e.g. $\mathrm{NO}_{2}$, radicals, and ions) reacted before reaching the light pipe, and therefore could not be detected. Other species, such as $\mathrm{N}_{2}$ and $\mathrm{H}_{2}$, do not absorb in the infrared region, and therefore were not detected. 


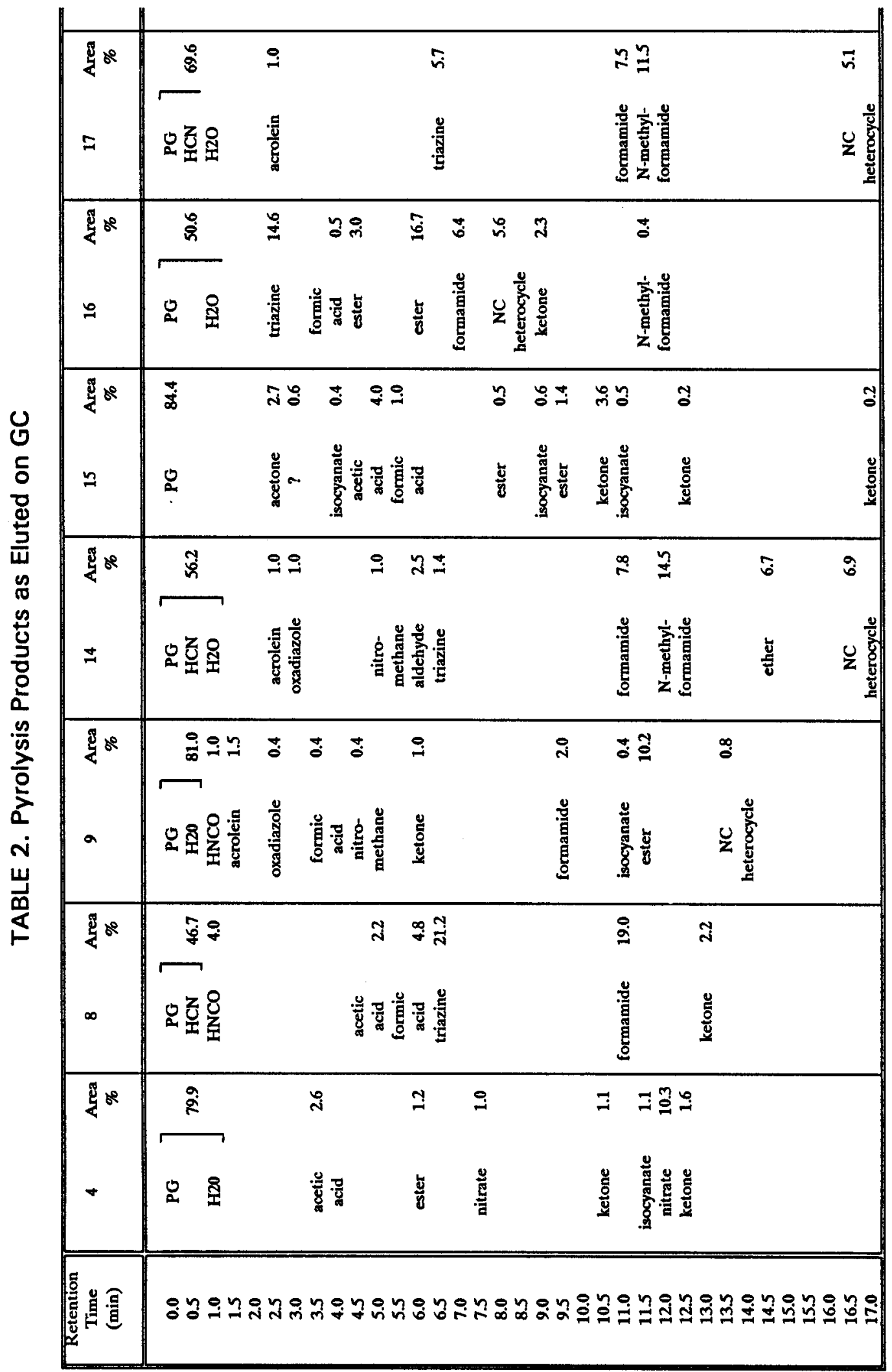




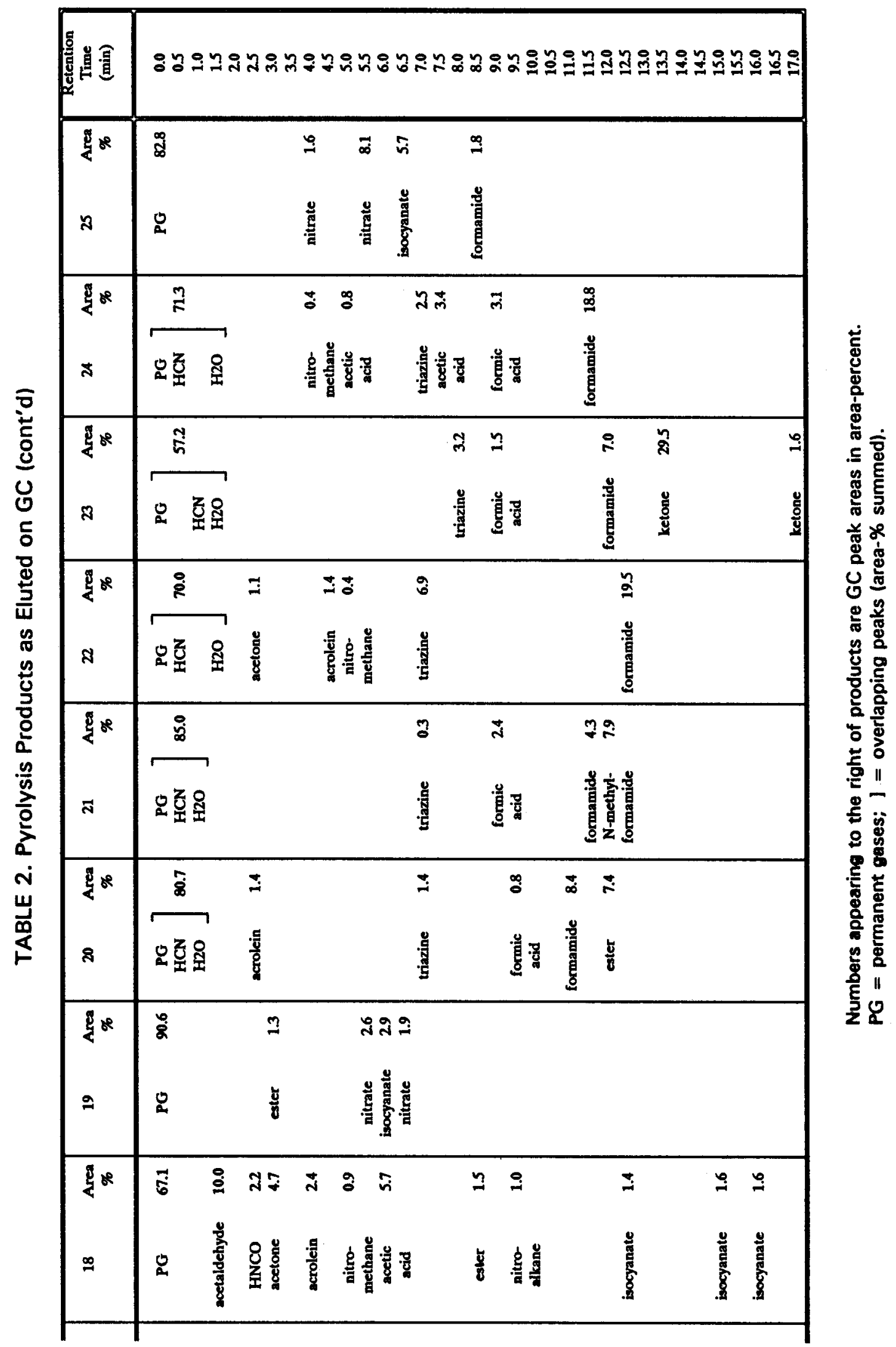


TABLE 3: Individual Permanent Gas Pyrolysis Products

\begin{tabular}{|c|cccccc|}
\hline $\begin{array}{c}\text { Sample } \\
\text { No. }\end{array}$ & CH4 & CH2O & CO2 & N2O & CO & NO \\
& \multicolumn{7}{|c|}{ (Normalized IR Absorbance) } \\
\hline 4 & & 0.14 & 0.45 & 0.37 & 0.01 & 0.03 \\
9 & 0.03 & & 0.43 & 0.38 & 0.06 & 0.09 \\
14 & 0.06 & & 0.37 & 0.43 & 0.06 & 0.08 \\
17 & 0.03 & & 0.38 & 0.45 & 0.04 & 0.09 \\
19 & 0.05 & 0.07 & 0.49 & 0.34 & 0.03 & 0.05 \\
20 & 0.04 & 0.41 & 0.07 & 0.09 \\
21 & 0.04 & & 0.40 & 0.40 & 0.07 & 0.09 \\
22 & 0.07 & & 0.38 & 0.41 & 0.06 & 0.09 \\
24 & 0.06 & & 0.39 & 0.42 & 0.06 & 0.08 \\
25 & & 0.16 & 0.45 & 0.32 & 0.02 & 0.05 \\
\hline
\end{tabular}

There are several striking differences between the pyrolysis product distributions of RDX and HMX formulations. Most are likely due to differences in pyrolysis temperatures. All RDX-based formulations were pyrolyzed at a set temperature that was $100^{\circ} \mathrm{C}$ lower than that for HMX-based formulations. This was done to compensate for the difference in oxidizer melting temperatures (i.e. $204^{\circ} \mathrm{C}$ for RDX and $280^{\circ} \mathrm{C}$ for $\mathrm{HMX}$ ). Since both RDX and $\mathrm{HMX}$ rapidly decompose at their melting points, $\mathrm{HMX}$ is almost $100^{\circ} \mathrm{C}$ higher than $\mathrm{RDX}$ when it actually melts.

\section{Selection of performance data for correlation with pyrolysis products}

Performance test results are given in Table 4. Examination of burn rate and impact sensitivity vs specific impulse indicates a strong correlation and suggest that these two measurements are thermodynamically controlled. First light and go-no go ignition times do not show such a correlation and are therefore not believed to be thermodynamically controlled, making them suitable choices for possible correlations with pyrolysis product distributions.

\section{Examination of go/no-go ignition times as a function of laser flux suggests that} samples can be divided into three groups. Group I is composed of samples 14, 22, 25, and 24 , and appears to experience relatively severe overdriven ignition [ref. 4] as evidenced by increasing go/no-go times with increasing laser flux. Group II is composed of samples 20, $21,4,9$, and 19 , and demonstrates less severe overdriven ignition. Group III, composed only of sample 17, does not appear to have a serious problem with overdriven ignition.

TABLE 4. Propellant Performance Test Results

\begin{tabular}{|c|c|c|c|c|c|c|c|c|c|c|c|}
\hline \multirow{3}{*}{ Sample } & \multirow{3}{*}{$\begin{array}{l}\text { Isp (a) } \\
(1 / s)\end{array}$} & \multirow{3}{*}{$\begin{array}{l}\text { Impact } \\
(\mathrm{cm})\end{array}$} & \multirow{3}{*}{$\begin{array}{c}\text { Burn Rate (b) } \\
(\mathrm{mm} / \mathrm{s})\end{array}$} & \multicolumn{8}{|c|}{ Ignition Times } \\
\hline & & & & \multicolumn{4}{|c|}{ Go/No-Go (ms) } & \multicolumn{4}{|c|}{ First Light (ms) } \\
\hline & & & & $60(c)$ & $100(c)$ & $150(c)$ & $200(c)$ & $60(c)$ & $100(c)$ & $150(c)$ & $200(c)$ \\
\hline 4 & 257.8 & 13.6 & 7.9 & 11.5 & 5.8 & 4.7 & 8.0 & 10.3 & 5.1 & 2.4 & 1.5 \\
\hline 8 & 235.4 & 30.2 & & & & & & & & & \\
\hline 9 & 257.0 & 15.6 & 7.6 & 9.9 & 5.1 & 3.4 & 6.1 & 8.9 & 4.2 & 1.5 & 0.6 \\
\hline 14 & 236.8 & 23.4 & 7.1 & 43.6 & 50.4 & 70.4 & 86.4 & 9.1 & 3.9 & 2.7 & 1.3 \\
\hline 15 & 221.4 & 33.9 & & & & & & & & & \\
\hline 16 & 213.9 & 41.6 & & & & & & & & & \\
\hline 17 & 214.6 & 25.7 & 3.6 & 22.4 & 22.6 & 17.1 & 11.1 & 7.8 & 4.9 & 2.4 & 1.5 \\
\hline 18 & 216.9 & 51.3 & & & & & & & & & \\
\hline 19 & 251.0 & 22.9 & 7.1 & 8.2 & 4.5 & 4.2 & 5.4 & 6.3 & 3.7 & 2.3 & 1.0 \\
\hline 20 & 251.3 & 21.9 & 7.9 & 10.2 & 7.3 & 10.8 & 17.7 & 6.7 & 3.8 & 2.2 & 1.2 \\
\hline 21 & 246.3 & 18.6 & 7.4 & 11.5 & 8.2 & 8.5 & 13.0 & 7.1 & 4.7 & 2.8 & 1.5 \\
\hline 22 & 243.1 & 17.0 & 6.9 & 15.8 & 20.5 & 33.2 & 53.2 & 7.0 & 5.1 & 3.2 & 1.3 \\
\hline 23 & 241.6 & 20.9 & & & & & & & & & \\
\hline 24 & 249.1 & 20.6 & 6.6 & 14.4 & 20.8 & 18.5 & 20.7 & 9.4 & 4.1 & 2.7 & 1.9 \\
\hline 25 & 248.8 & 21.3 & 6.4 & 10.6 & 13.1 & 21.6 & 29.5 & 8.0 & 3.9 & 2.0 & 1.9 \\
\hline
\end{tabular}

c) All ignition times at specified laser flux $\left(\mathrm{cal} / \mathrm{cm}^{2} \mathrm{~s}\right)$, measured at $250 \mathrm{psi}$. 


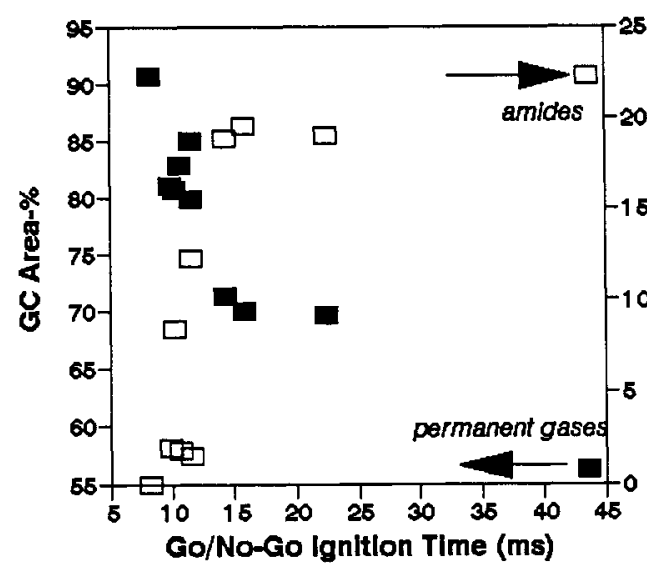

Fig. 1. Correlation plot for permanent gas and amide pyrolysis products vs. go/no-go ignition time.

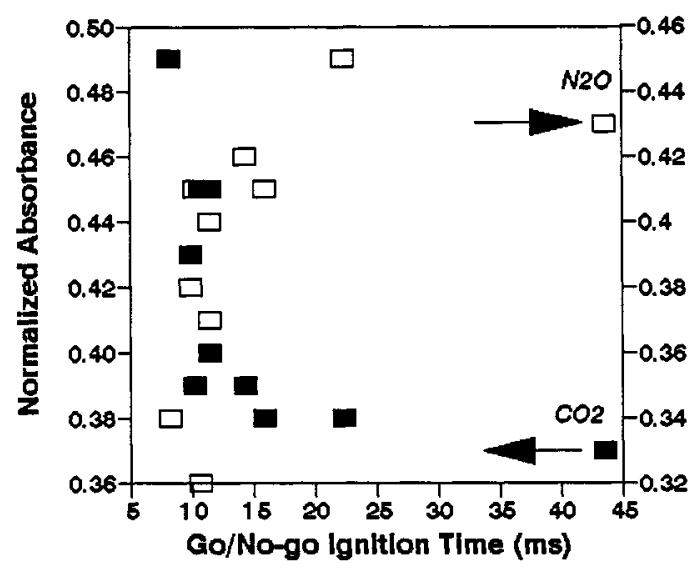

Fig. 2. Correlation plot for $\mathrm{CO}_{2}$ and $\mathrm{N}_{2} \mathrm{O}$ pyrolysis products vs. go/no-go ignition time.

Pyrolysis-product/ignition-data correlations

To identify correlations, several techniques and tools were used. These include simple visual examination of P-GC-FTIR data in formats similar to those in Table 2, as well as a multitude of plots generated by spreadsheet programs and multivariate analysis programs. Possible correlations for all pyrolysis products vs. all ignition data were explored. Several correlations were observed, the best of these were the correlations between the total permanent gas products and total amides vs. go/no-go ignition time at the lowest laser flux (Fig 1). Correlations at higher laser fluxes were also observed, but due to overdriven ignition, these correlations were inferior to that observed at the lowest laser flux.

$\mathrm{N}_{2} \mathrm{O}$ vs go/no-go ignition time were also observed (Fig. 2). In general it may be said that formulations pyrolyzing most efficiently, i.e. generating the "smallest" products have the shortest go/no-go ignition times. One possible explanation for this observation is that more efficient pyrolysis at the surface of the propellant results in a higher surface temperature, permitting the establishment of the thermal profile necessary for ignition.

The absense of samples with appropriate radiative ignition measurement times has made it difficult to determine the validity of the observed correlations for prediction of go/no-go ignition times for samples other than those examined in this investigation. Several "rough" predictions of ignition time have held true (e.g. that samples will have very short ignition times or will have difficulty igniting). (ref. 8)

In searching for explanations as to why certain samples with very similar formulations exhibit dissimilar ignition times, the possibility that certain pyrolysis products might preferentially absorb at the wavelength of the laser $(10.6 \mu \mathrm{m})$ was considered. Examination of the infrared spectrum of all pyrolysis products (ref. 9) indicated that such preferential absorption was not significant.

Effect of plasticizer on pyrolysis product distribution

Inspection of the composition of samples in Groups I, II, and III reveals a correlation between plasticizer type and the tendency of the propellant to experience overdriven ignition, i.e. Group I contains mostly TMETN-plasticized formulations, while Group II contains mostly BTTN-plasticized formulations. Trends in pyrolysis product distributions also appear to be related to the plasticizer used in the formulation. For example, HMXbased formulations plasticized with BTTN generated less triazine than those plasticized with 
TMETN. Plasticized RDX-based formulations were the only samples that gave no triazine (except for samples 15 and 18, which contained no nitramine). Also, plasticized RDX formulations were the only samples to generate formaldehyde but not methane. With respect to permanent gas yields, it was observed that unplasticized formulations generated the lowest amounts of permanent gases; BTTN-containing formulations generated larger amounts of permanent gases than did TMETN-containing formulations. Based on these observations, it appears that the nitrate ester plasticizers or their decomposition products may play a special role in the thermal decomposition of these propellant formulations. To gain insight into this matter, the pyrolysis products of BTTN and TMETN were examined (not shown). It was found that in addition to $\mathrm{CO}_{2}, \mathrm{~N}_{2} \mathrm{O}, \mathrm{CO}$, and $\mathrm{NO}$, both plasticizers yield a relatively large amount of formaldehyde as well as several nitrate ester fragments. BTTN was observed to generate more formaldehyde and other permanent gases than did TMETN. Which, if any, of the plasticizer pyrolysis products might act a catalyst has not been determined, though formaldehyde has been reported to catalyze the thermal decomposition of nitramines. (refs 5-7) Further investigation into this matter is currently in progress.

\section{CONCLUSION}

The correlations identified in this investigation provide information regarding the "chemical" cause of overdriven ignition, as well as a means for predicting go/no-go ignition times. It was observed that samples having relatively long ignition times pyrolyzed less efficiently (i.e. generated less permanent gases and more "large" fragments) than did samples having relatively short ignition times. It is suggested that more efficient pyrolysis results in a higher surface temperature due to larger heat releases at the propellant surface. The higher surface temperature then supports the establishment of the thermal profile necessary for ignition to occur.

Samples experiencing relatively severe overdriven ignition were observed to generate more amides and isocyanates, and less esters, than samples experiencing less severe overdriven ignition.

With respect to the effect of propellant ingredients on ignition times and pyrolysis product distributions, it was observed that both depend heavily on the plasticizer used in the propellant formulation and it is proposed that formaldehyde generated by pyrolyzed plasticizer acts as a catalyst in the thermal decomposition process.

\section{Acknowledgement}

We thank Dr. Rena Y. Yee of Naval Weapons Center, China Lake, California, for kindly providing all samples and performance test results.

\section{REFERENCES}

1. R.A. Fifer, in Fundamentals of Solid Propellant Combustion, K.K. Kuo and M. Summerfield Eds. Progress in Astronautics and Aeronautics, AIAA, NY, 90 (1984).

2. M.A. Schroeder, BRL-TR-2659, (1985).

3. M.A. Schroeder, Proceedings of the $25^{\text {th }}$ JANNAF Combustion Meeting, CPIA Pub. 498, Vol. III, 421-431 (1988).

4. T.J. Ohlemiller, L.H. Caveny, L. DeLuca, and M. Summerfield, Proceedings of the $14^{\text {th }}$ Symposium (International) on Combustion, The Combustion Institute, 1297-1307 (1972).

5. D.J. Cosgrove and A.J. Owen, Comb. Flame., 22 19-22 (1974).

6. J.J. Batten, Aust. J. Chem., 24, p.945 (1971).

7. J.J. Batten, Aust. J. Chem., 24, p.2025 (1971).

8. R.A. Pesce-Rodriguez, unpublished results (1992).

9. R.A. Pesce-Rodriguez, F.J. Shaw, and R.A. Fifer, BRL-TR-3288, Ballistic Research Laboratory, Aberdeen Proving Ground, MD (1991) 